\title{
Certificación de un material de referencia de carne en base a un estudio de colaboración
}

\section{Certification of a meat reference material based on a collaborative study}

Salazar Arzate, Claudia Marcela (7), Regalado, Laura (7), Pazos, Alberto ${ }^{(1)}$, Vivino, Enrique (1), García, Vania (2), Pantoja, María Rosa (2), Rocha, Eliana (2), Vacaflor, Patricia (2), Casiano, Rodrigo ${ }^{(3)}$, Cucatti, Mónica Regina (3), Miyagushu, Luciana (3), Sousa, Renata ${ }^{(3)}$, Uekane, Thais ${ }^{(3)}$, Gonçalves de Melo, Monique ${ }^{(3)}$, Acevedo, Xiomara ${ }^{(4)}$, Jimenez, Elizabeth ${ }^{(4)}$, Jimenez, Linda Patricia ${ }^{(4)}$, Rivera, Myriam Jessel ${ }^{(4)}$, Torres, Pedro Hernán ${ }^{(4)}$, Larrea, Elena ${ }^{(5)}$, Navas, Lucía (5), Silva, Susana (5), Phillips, Andrene ${ }^{(6)}$, Random, Dwight ${ }^{(6)}$, Acco, Steve ${ }^{(8)}$, Ramos, Ana ${ }^{(8)}$, Uribe, Christian $^{(8)}$, Ticona, Galia ${ }^{(8)}$, Toro, Cristina ${ }^{(8)}$, Francini, Lorena ${ }^{(9)}$, Reinares, Rosana ${ }^{(9)}$

(1) Instituto Nacional de Tecnología Industrial, INTI, Argentina - ${ }^{(2)}$ Instituto Boliviano de Metrología, IBMETRO, Bolivia (3) Instituto Nacional de Metrología, Normalización y Calidad Industrial, INMETRO, Brasil - (4) Superintendencia de Industria y Comercio, SIC, Colombia - (5) Instituto Ecuatoriano de Normalización, INEN, Ecuador - (6) Bureau of Standards Jamaica, BSJ, Jamaica - (7) Centro Nacional de Metrología, CENAM, México - ${ }^{(8)}$ Instituto Nacional de Defensa de la Competencia y de la Protección de la Propiedad Intelectual, Servicio Nacional de Metrología, INDECOPI, Perú ${ }^{(9)}$ Laboratorio Tecnológico del Uruguay, LATU, Uruguay.

Contacto: csalazar@cenam.mx

Recibido: 25/1/2011 - Aprobado: 28/9/2012

\section{Resumen}

Mediante un proyecto de colaboración, se llevaron a cabo estudios de comparación para mejorar la capacidad de medición de los laboratorios participantes, apoyándolos en la producción, caracterización y distribución de materiales de referencia del sector agroalimentario. EI proyecto fue planteado en cuatro etapas anuales (leche, agua, carne y granos). La tercera etapa en específico tuvo el objetivo de cuantificar y certificar el contenido de los parámetros nutrimentales (nitrógeno, grasa, sodio y potasio) de un lote candidato a Material de Referencia Certificado (MRC) de carne bovina enlatada. Dicho estudio fue realizado en colaboración entre varios Institutos Nacionales de Metrología (INM) y/o laboratorios colaboradores, los cuales, una vez que identificaron las posibles causas de variabilidad o de sesgo en las mediciones, así como las oportunidades de mejora, lograron la certificación del material de carne bovina. EI MRC fue distribuido entre los participantes para cubrir las necesidades de la industria alimentaria de productos cárnicos y laboratorios de prueba de sus respectivos países.

Palabras clave: Material de referencia, Material de Referencia Certificado, trazabilidad, incertidumbre.

\begin{abstract}
$\underline{\text { Abstract }}$
Through a collaborative project, comparison studies were carried out to improve measurement capabilities of participating laboratories, supporting them to produce, characterize and distribute reference materials in the food sector. The project was planned in four annual stages (milk, water, meat and grains). The third stage aimed specifically to quantify and certify the nutritional content of the parameters (nitrogen, fat, sodium and potassium) of a batch candidate as Certified Reference Material (CRM) of canned beef. This study was conducted in collaboration between several National Metrology Institutes (NMIs) and/or collaborating laboratories, which, once identified the possible causes of variability or bias in the measurements, as well as the opportunities of improvement, achieved the certification of the material beef. The CRM was distributed among the participants to cover the needs of the food industry of meat products and testing laboratories in their respective countries.

Keywords: Reference material, Certified Reference Material, traceability, uncertainty.
\end{abstract}

\section{Introducción}

Dentro de la industria alimenticia, los parámetros de análisis más comunes en la evaluación de la calidad de los productos son conocidos como proximales y constituyen parámetros de medición rutinaria. Sin embargo, la disponibilidad de Materiales de Referencia Certificados (MRC) afines a estos es aún limitada en el mercado. Atender la necesidad de contar con Materiales de Referencia (MR) que contribuyan a mantener e incluso mejorar la calidad de medición de los laboratorios analíticos es uno de los mayores objetivos de los
Institutos Nacionales de Metrología (INM), los cuales son, además, responsables de establecer la trazabilidad de las mediciones analíticas y su comparabilidad.

Con este antecedente y la finalidad de mejorar la disponibilidad y el desarrollo de MR, así como de fomentar la colaboración entre laboratorios de diferentes regiones, se generó mediante el apoyo de la Organización de Estados Americanos (OEA) un proyecto para producir, certificar y compartir MRC requeridos para asegurar la calidad de alimentos de interés común, en este caso, carne. El proyecto se realizó bajo el esquema de comparación y contó con la 
participación y experiencia de instituciones dedicadas a la certificación de materiales.

Para esta tarea, un lote de 1.000 unidades de un candidato a MR de carne tipo paté contenida en latas de aluminio de aproximadamente 90 $\mathrm{g}$ fue producido y distribuido por el Instituto Nacional de Tecnología Industrial (INTI) de Argentina al resto de los participantes, quienes recibieron sub lotes de 100 unidades cada uno.

El laboratorio coordinador del proyecto estuvo a cargo de evaluar la homogeneidad del contenido de los parámetros a certificar -acordados previamente-, nitrógeno, grasa, potasio y sodio, por tratarse de mediciones de control en los laboratorios de prueba.

Previo al proceso de certificación y con el objetivo de demostrar competencia técnica y obtener a su vez la mayor consistencia en los resultados -sobre todo para los parámetros dependientes del proceso operacional de medición, tales como nitrógeno y grasa- fue necesaria la revisión y "armonización” tanto de métodos como de prácticas de laboratorio llevadas a cabo por cada participante.

Conviene recordar que para los parámetros dependientes del procedimiento operacional una práctica comúnmente aceptada para la certificación de estos analitos son los estudios colaborativos, en los cuales, por medio de una evaluación de la consistencia de los resultados y la aplicación de conceptos estadísticos, es posible determinar los valores representativos de las propiedades del material en cuestión. Por otro lado, para los parámetros independientes del método se aplican directamente los conceptos de trazabilidad hacia los resultados de medición con mayor jerarquía metrológica, cuya incertidumbre sea la más pequeña que se pueda lograr.

Originalmente, se estableció que las incertidumbres máximas esperadas al final de las mediciones de proteína y grasa serían de 5 y $7 \%$, respectivamente; sin embargo, debido a que en los estudios de comparación realizados durante la etapa de leche los valores de incertidumbre fueron mayores, se estableció que para este ejercicio serían de 7 y $8 \%$.

En este trabajo se describen las consideraciones hechas por los laboratorios para llegar a definir los valores de certificación obtenidos y expresados para el MR de carne, con base en el estudio de comparación realizado entre los participantes del proyecto.

\section{Materiales y Métodos}

En cuanto a los métodos de medición, existen distintas prácticas para la evaluación de la calidad de los productos cárnicos entre los países participantes de este estudio. En la mayoría de los casos, son dictadas por la legislación de cada país, de modo que varían de región a región. Dichas diferencias pueden ser consultadas en las Tablas 11 a 14 del artículo.

En ese sentido, el proyecto para la caracterización del candidato a MR de carne se planteó en las siguientes fases:

1. Medición de muestras de carne y muestras de un MR con métodos habituales.

2. Armonización de métodos y prácticas de laboratorio.

3. Realización de nuevas mediciones de muestras de carne y control utilizando métodos armonizados.

4. Certificación del candidato a MR.

Durante la fase de "armonización", los participantes se abocaron a la tarea de identificar las variables importantes que se debían controlar durante los procedimientos de cuantificación de cada uno de los parámetros en análisis. Asimismo, acordaron establecer, en la medida de lo posible, las condiciones de trabajo y realizar cada vez nuevas mediciones con el objetivo de poder certificar el lote de carne bajo el esquema de colaboración original. Para este fin se empleó un MRC proporcionado por el Centro Nacional de Metrología (CENAM), que consistió en una mezcla de pollo con vegetales y que se usó de modo paralelo como control de medición.

Se realizó nuevamente el estudio de homogeneidad de los contenidos de nitrógeno, grasa, sodio y potasio con las mediciones reportadas con los métodos armonizados mediante análisis de varianza (ANOVA). El número de muestras consideradas fue diferente para cada parámetro.

Para las mediciones de sodio y potasio se emplearon los métodos basados en espectrometría de absorción atómica (EAAF), emisión atómica con plasma acoplado inductivamente (ICP-AES) o espectrometría de masas con plasma acoplado inductivamente (ICPMS), y también cromatografía de líquidos de alto desempeño (HPLC).

Para algunos de los parámetros fue necesario que ciertos laboratorios realizaran varias veces las mediciones, ya que las reportadas originalmente presentaban sesgo y/o dispersión y, por lo tanto, no se consideraban adecuadas para incluirlas en el proceso de certificación de los parámetros.

A continuación se muestran los resultados que se obtuvieron para cada parámetro. Para cada uno de los analitos, la asignación del valor certificado y la estimación de su incertidumbre asociada se realizó de forma individual.

\section{Resultados}

\section{Nitrógeno}

La homogeneidad del lote fue evaluada mediante análisis de varianza (ANOVA). Se consideraron un total de 68 muestras, cada una con tres réplicas. Las muestras fueron seleccionadas de forma aleatoria por los laboratorios participantes. El estudio indicó que el contenido de nitrógeno en el candidato a material de referencia de carne era homogéneo, por lo que se continuó con la certificación en conjunto.

En la Figura 1 se presentan los valores medidos para cada réplica, del lado derecho, el valor promedio y las barras de incertidumbre reportados por cada participante. La línea sólida representa el valor promedio del total de los resultados reportados y las líneas punteadas, dos veces la desviación estándar.

\section{Grasa}

Los resultados de grasa están contenidos en la Figura 2. Como se observa, los laboratorios 2 y 4 presentaban valores sesgados que contribuían a una desviación estándar grande, que podía ser disminuida considerablemente si estos eran corregidos o eliminados. Igualmente, los resultados de los laboratorios 7 y 8 afectaron la evaluación de la calidad de las muestras.

La eliminación de los resultados sesgados con respecto al valor medio global afectaba directamente al valor medio del total de valores (Tabla 1), pero sobre todo a la desviación estándar relativa, la cual cambiaba de $2,4 \%$ a $0,6 \%$ (Tabla 5 ).

Por consiguiente, se recomendó a los laboratorios mencionados repetir sus mediciones y a los identificados con los números 6,7 y 8 revisar sus resultados a fin de encontrar mejoras que pudieran reducir la desviación estándar.

Los resultados obtenidos finalmente son los presentados en la Figura 3. 


\begin{tabular}{|c|c|c|}
\hline & Todos las muestras & $\begin{array}{c}\text { Excluyendo a los } \\
\text { laboratorios 2 y 4 }\end{array}$ \\
\hline Media del grupo & $20,01 \mathrm{~g} / 100 \mathrm{~g}$ & $20,25 \mathrm{~g} / 100 \mathrm{~g}$ \\
\hline Desviación estándar de la media & $0,49 \mathrm{~g} / 100 \mathrm{~g}$ & $0,13 \mathrm{~g} / 100 \mathrm{~g}$ \\
\hline DER & $2,45 \%$ & $0,64 \%$ \\
\hline
\end{tabular}

Tabla 1. Comparación de valores reportados para grasa.

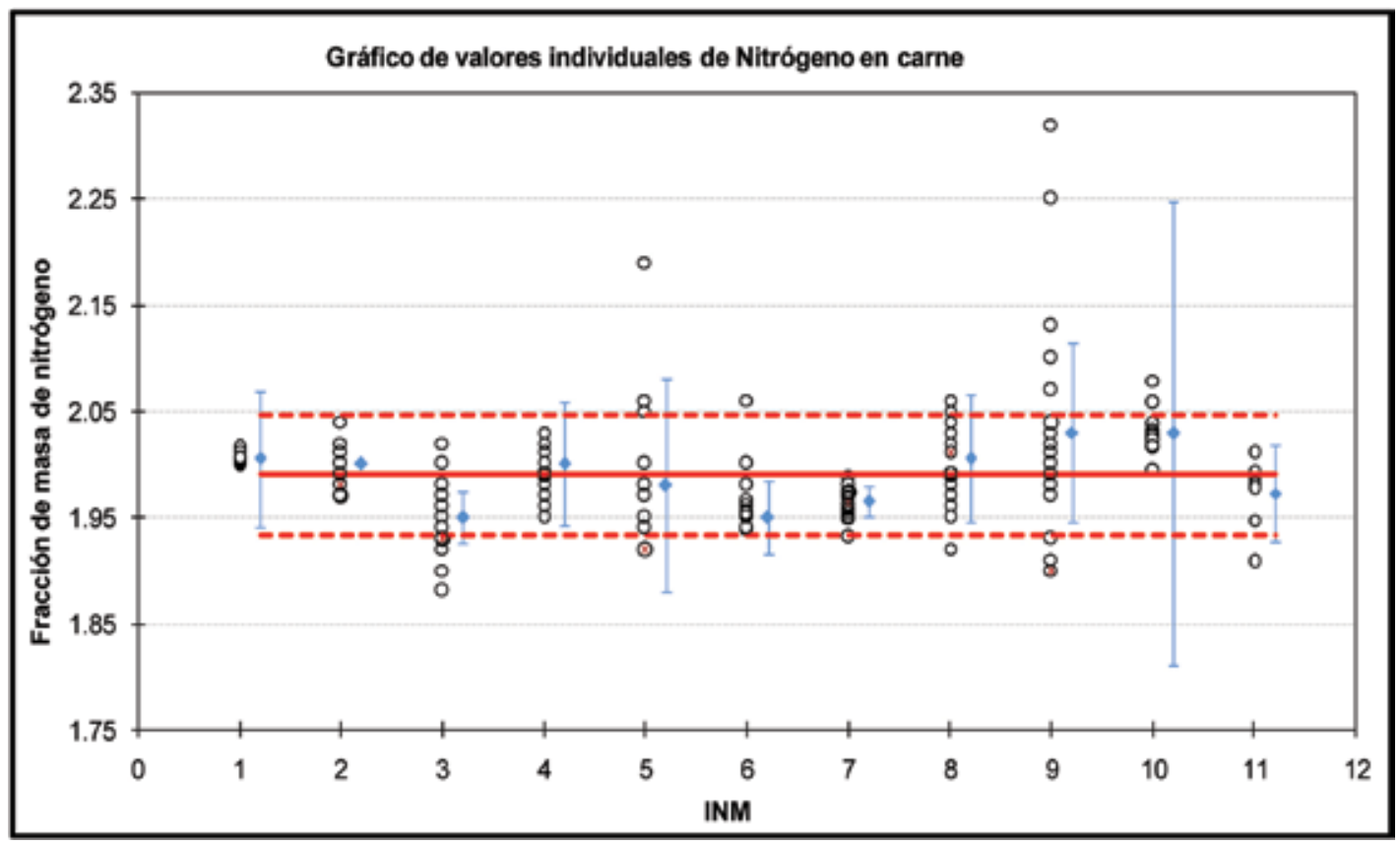

Figura 1. Resultados de la medición de nitrógeno en carne.

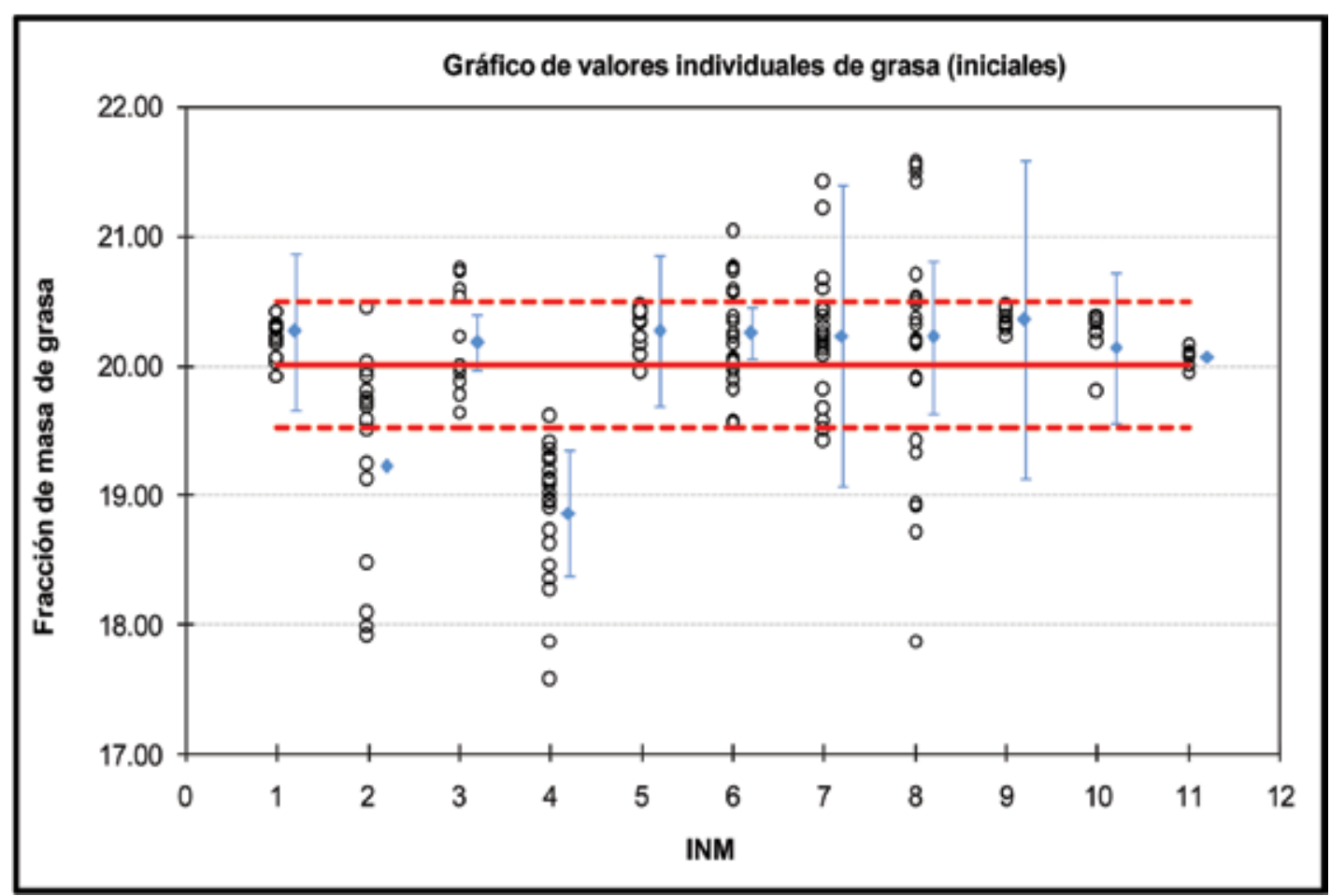

Figura 2. Resultados iniciales de la medición de grasa en carne. 


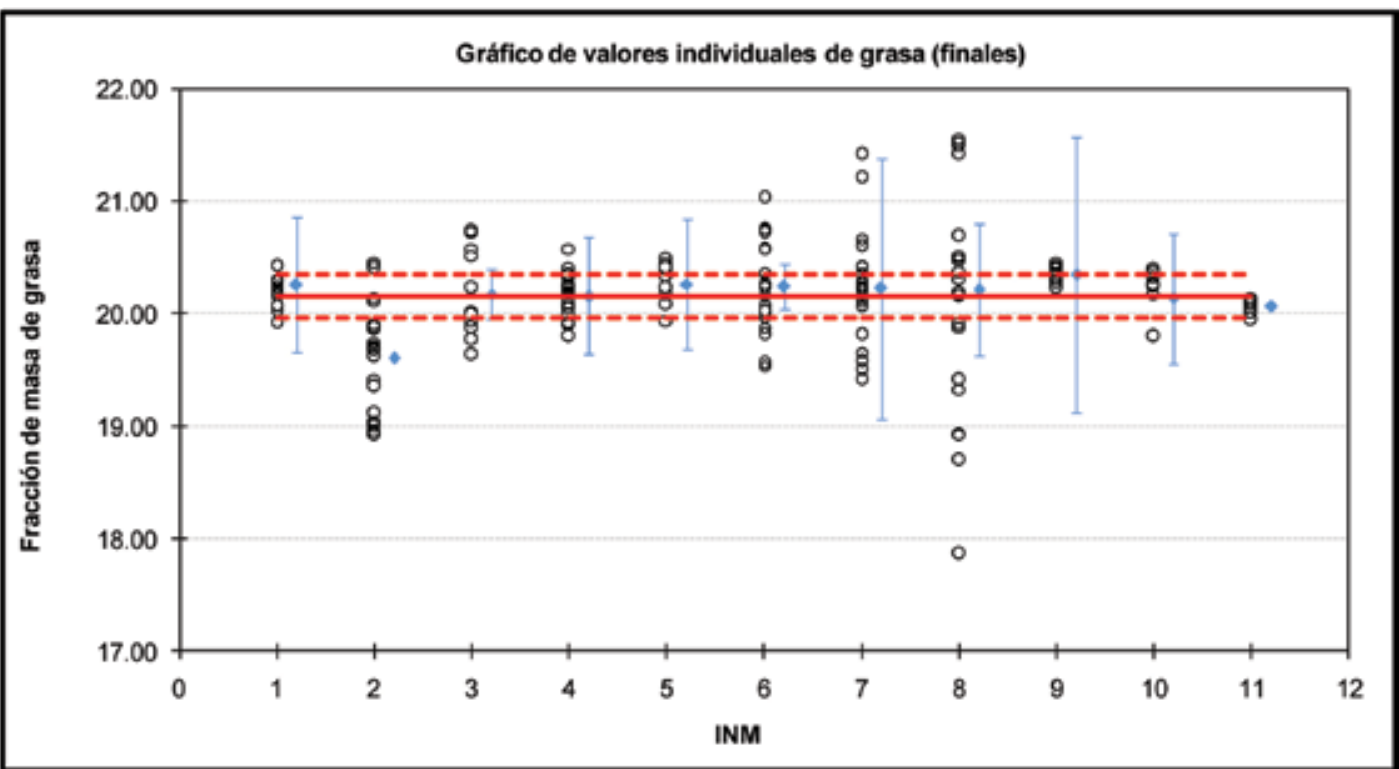

Figura 3. Resultados finales de la medición de grasa en carne.

\section{Sodio}

Los resultados de sodio se expresan en la Figura 4. Para dicho analito los resultados de los laboratorios 1, 2, 3, 5, 6 y 7 fueron obtenidos por EAAF, mientras que los resultados de los laboratorios 9 y 11 por ICP-AES, el 10 proviene de ICP-MS y los de los laboratorios 4 y 8 fueron obtenidos por HPLC.

Conforme a los resultados, se les recomendó a los laboratorios 2 y 7 volver a medir para mejorar la dispersión entre sus mediciones y/o el sesgo respecto a la mayoría. Los resultados que fueron reportados se muestran en la Figura 5.

\section{Potasio}

Los resultados de potasio se presentan en la Figura 6. Al igual que en el caso de sodio, los resultados de los laboratorios 1, 2, 3, 5, 6, y 7 fueron obtenidos por EAAF, mientras que los resultados de los laboratorios 9 y 11 por ICP-AES, el 10 proviene de ICP-MS y los de los laboratorios 4 y 8 fueron obtenidos por HPLC.

En este caso, se le solicitó al laboratorio 7, entre otros, la revisión de sus resultados. Los finales son presentados en la Figura 7.

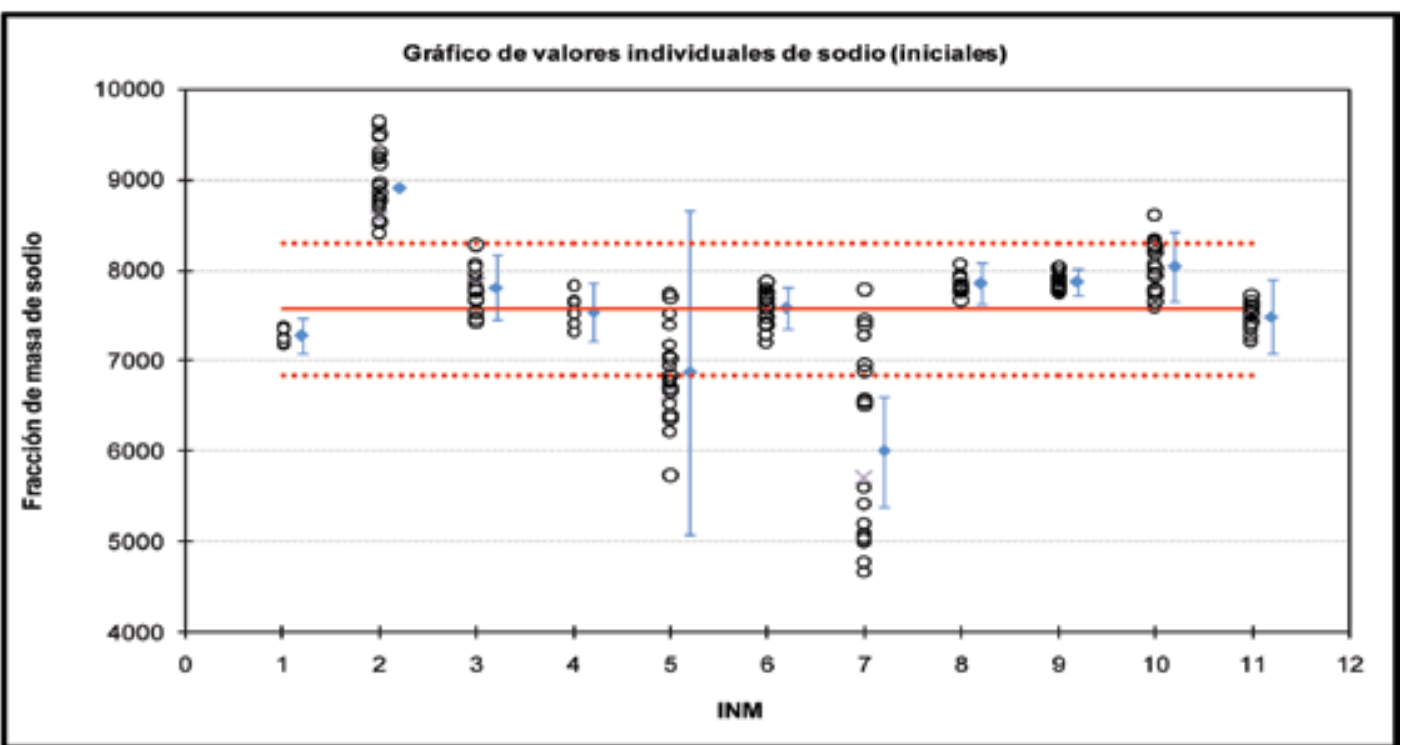

Figura 4. Resultados iniciales de la medición de sodio en carne. 


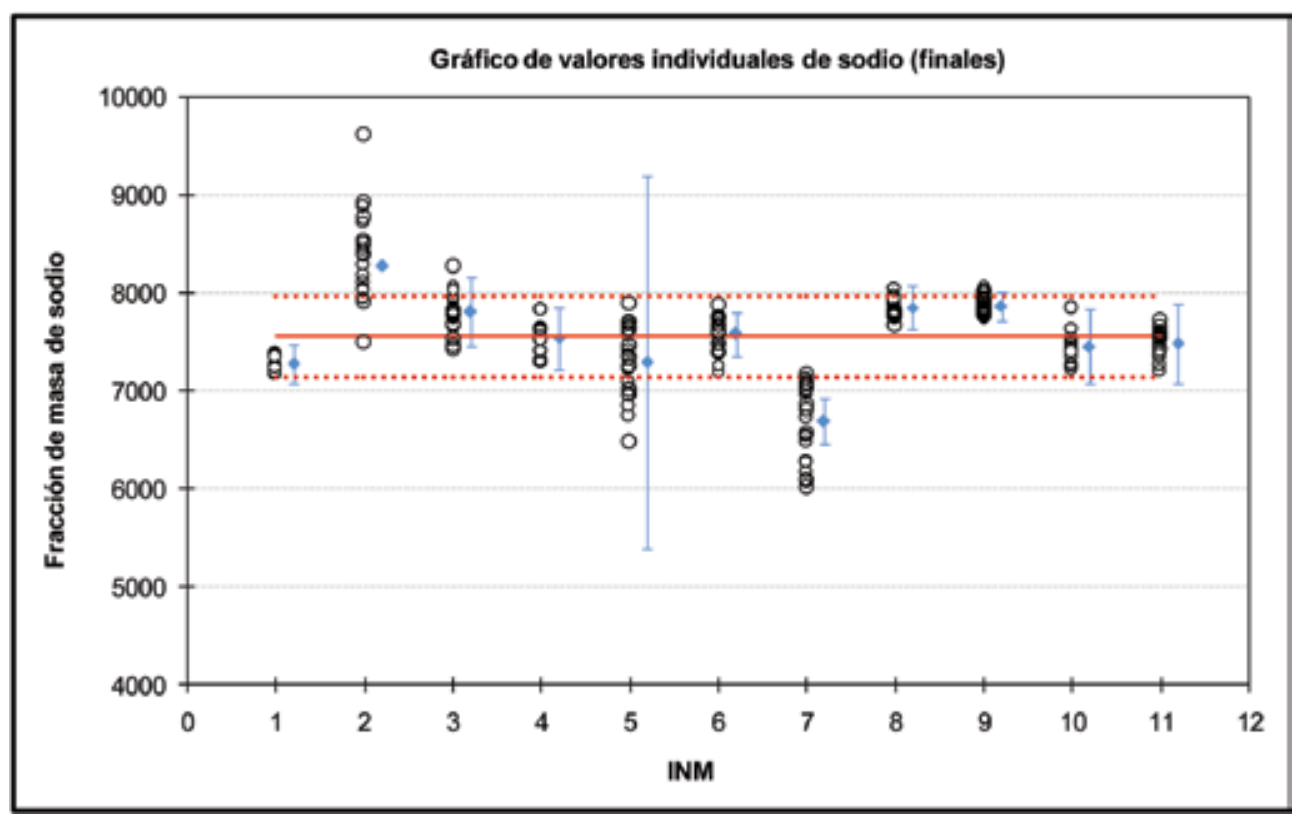

Figura 5. Resultados finales de la medición de sodio en carne.

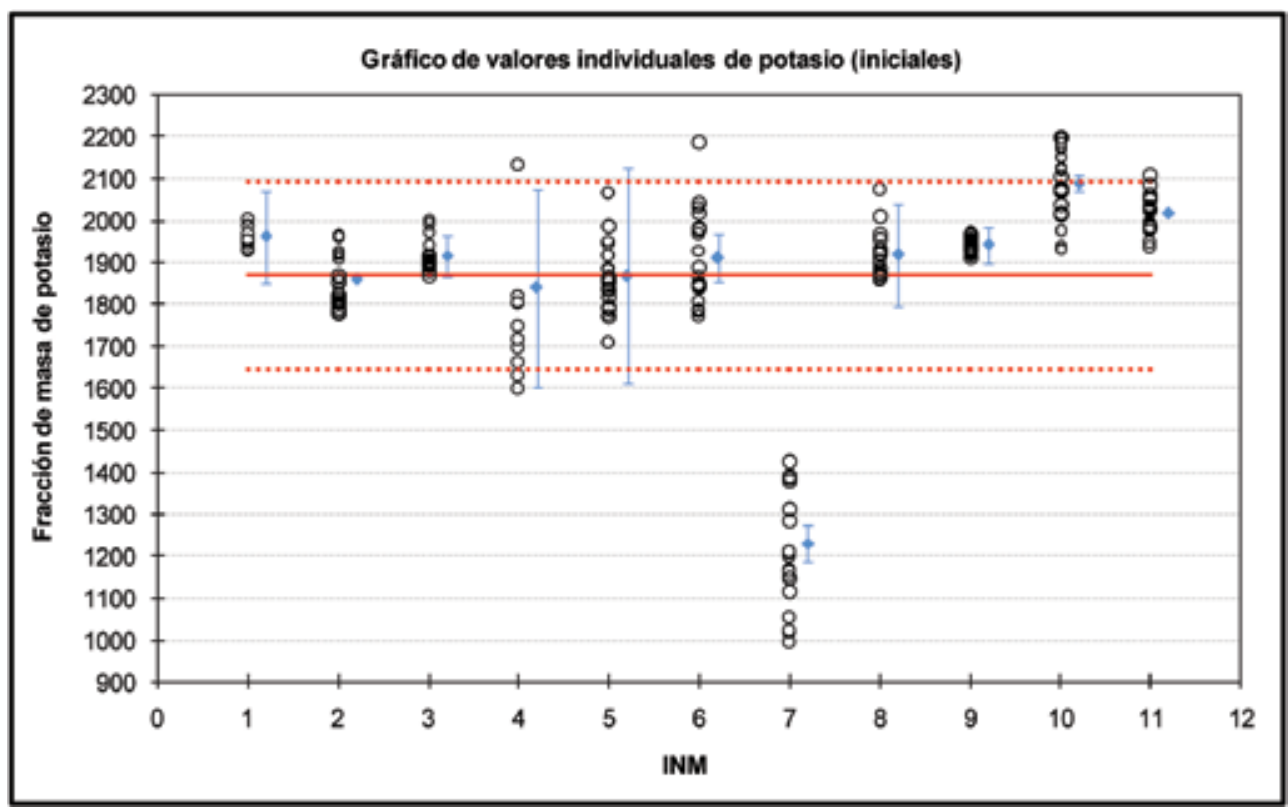

Figura 6. Resultados iniciales de la medición de potasio.

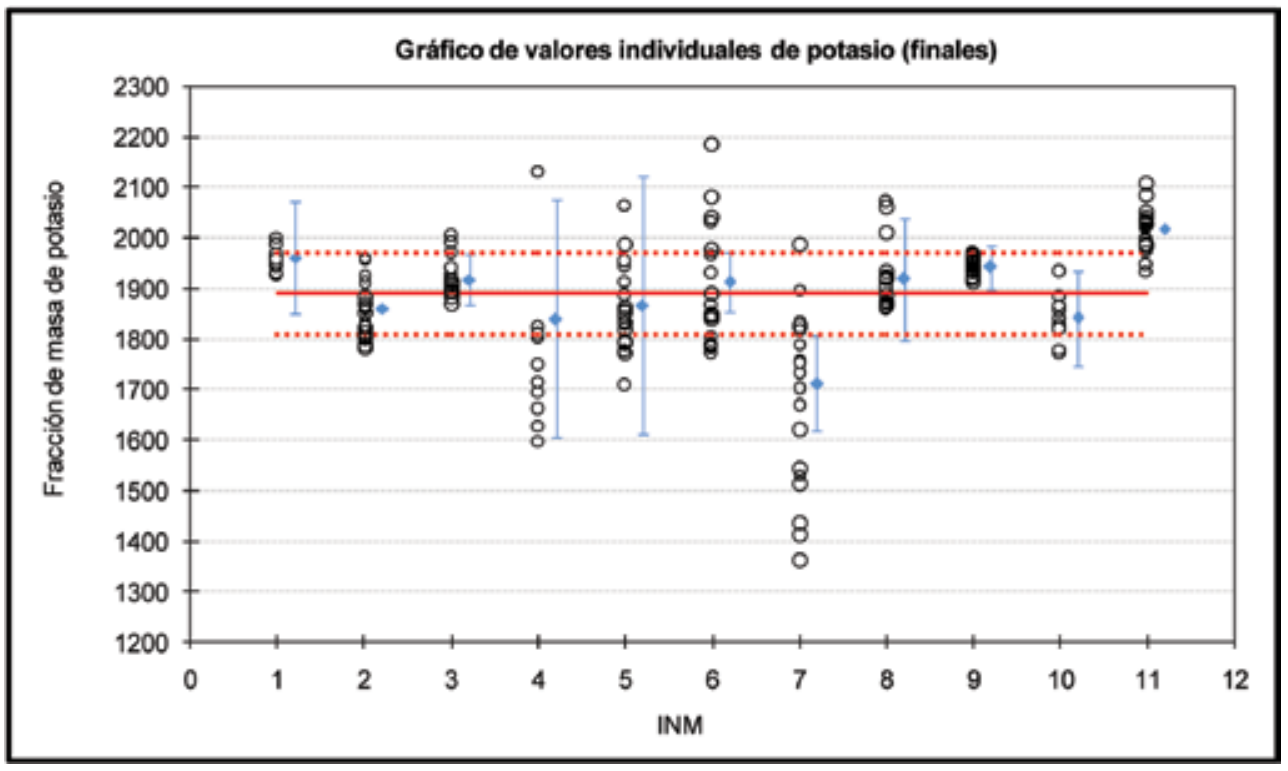

Figura 7. Resultados finales de la medición de potasio en carne. 


\section{Discusión}

En general, el ejercicio de armonización con un MRC permitió identificar las oportunidades de mejora para cada laboratorio, incrementando la calidad de los resultados para las muestras de candidatos a MR en cuanto a la consistencia, tal y como se observa en la Tabla 2.

\begin{tabular}{|l|c|c|c|c|}
\hline \multirow{2}{*}{ Mensurando } & \multicolumn{2}{|c|}{ Antes de la armonización } & \multicolumn{2}{c|}{ Después de la armonización } \\
\cline { 2 - 5 } & Promedio & $\begin{array}{c}\text { Desviación Estándar } \\
\text { Relativa (\%) }\end{array}$ & Promedio & $\begin{array}{c}\text { Desviación Estándar } \\
\text { Relativa (\%) }\end{array}$ \\
\hline Nitrógeno & $1.97 \mathrm{~g} / 100 \mathrm{~g}$ & 4,5 & $1,99 \mathrm{~g} / 100 \mathrm{~g}$ & 3,0 \\
\hline Grasa & $19.83 \mathrm{~g} / 100 \mathrm{~g}$ & 5,6 & $20,01 \mathrm{~g} / 100 \mathrm{~g}$ & 2,4 \\
\hline Sodio & $7456 \mathrm{mg} / \mathrm{kg}$ & 13,7 & $7567 \mathrm{mg} / \mathrm{kg}$ & 9,6 \\
\hline Potasio & $1814 \mathrm{mg} / \mathrm{kg}$ & 17,5 & $1847 \mathrm{mg} / \mathrm{kg}$ & 11,5 \\
\hline
\end{tabular}

Tabla 2. Resultados obtenidos antes y después de la fase de armonización.

Los ajustes realizados en la metodología armonizada de Kjeldahl permitieron obtener resultados congruentes. Dichos ajustes consistieron principalmente en la estandarización de la normalidad del ácido utilizado. El valor certificado obtenido fue de $(1,99 \pm 0,078) \mathrm{g} / 100 \mathrm{~g}$ con una incertidumbre estándar relativa de 3,9\%, con lo que se logró el objetivo planteado para el esquema de colaboración.

Respecto a la grasa, al comparar las Figuras 2 y 3 puede apreciarse la considerable mejora que tuvo el laboratorio 4 al realizar ajustes en su procedimiento. En cuanto al resultado del laboratorio 2, a pesar de que aumentó el contenido de grasa extraída, se mantuvo un sesgo respecto al resto de los valores, por lo que se consideró pertinente la eliminación de sus datos para el estudio de ANOVA y la emisión del valor de referencia “más cercano al verdadero". El valor medio de grasa resultó de 20,16 g/100g con una desviación estándar relativa de $1 \%$.

A la par de la revisión de los resultados de grasa, surgió entre los participantes el cuestionamiento del tipo de grasa cuantificada, debido a los tres procesos utilizados: grasa libre por soxhlet, grasa total por hidrólisis ácida y grasa total por soxhlet e hidrólisis ácida.

Por tal razón, en paralelo al estudio en conjunto y dadas la clasificación de la carne, la normativa oficial de cada región y las experiencias respecto a su determinación, se analizaron los resultados de acuerdo a las metodologías y al "tipo de grasa" extraída. Los valores obtenidos según las clasificaciones se presentan a continuación.

a) Grasa libre, extracción por Soxhlet. Fue determinada por los laboratorios 1, 3, 4, 6, 8 y 10. Para este grupo, el valor promedio fue de $19,97 \mathrm{~g} / 100 \mathrm{~g}$ con una desviación estándar de $0,55 \mathrm{~g} / 100 \mathrm{~g}$.

\begin{tabular}{|c|c|c|}
\hline Laboratorio & $\begin{array}{c}\text { Valor obtenido } \\
(\mathbf{g} / \mathbf{1 0 0} \mathbf{g})\end{array}$ & $\begin{array}{c}\text { Incertidumbre } \\
(\mathbf{g} / \mathbf{1 0 0 g})\end{array}$ \\
\hline 1 & 20,27 & 0,60 \\
\hline 3 & 20,18 & 0,22 \\
\hline 4 & 18,86 & 0,49 \\
\hline 6 & 20,25 & 0,20 \\
\hline 8 & 20,22 & 0,59 \\
\hline 10 & 20,07 & 0,11 \\
\hline Promedio & 19,97 & \\
\hline
\end{tabular}

Tabla 3. Resultados para grasa libre.

b) Grasa total, extracción con solvente previa hidrólisis ácida. Los resultados reportados por los laboratorios 5, 7 y 9 corresponden a este método.

En este caso, el promedio del grupo fue de 20,28 con una desviación estándar de 0,06 g/100 g.

\begin{tabular}{|c|c|c|}
\hline Laboratorio & $\begin{array}{c}\text { Valor obtenido } \\
(\mathbf{g} / \mathbf{1 0 0 g})\end{array}$ & $\begin{array}{c}\text { Incertidumbre } \\
(\mathbf{g} / \mathbf{1 0 0 g})\end{array}$ \\
\hline 5 & 20,27 & 0,58 \\
\hline 7 & 20,23 & 1,16 \\
\hline 9 & 20,35 & 1,23 \\
\hline Promedio & 20,28 & \\
\hline
\end{tabular}

Tabla 4. Resultados de grasa total por hidrólisis ácida.

c) Grasa total, extraída mediante soxhlet, previa hidrólisis. Los resultados reportados por los laboratorios 2 y 10 corresponden a este método. Para el grupo el promedio fue de 19,68 con una desviación estándar de 0,65 g/100g. 


\begin{tabular}{|c|c|c|}
\hline Laboratorio & $\begin{array}{c}\text { Valor obtenido } \\
(\mathbf{g} / \mathbf{1 0 0 g})\end{array}$ & $\begin{array}{c}\text { Incertidumbre } \\
(\mathbf{g} / \mathbf{1 0 0 g})\end{array}$ \\
\hline 2 & 20,14 & 0,58 \\
\hline 10 & 19,22 & - \\
\hline Promedio & 19,68 & \\
\hline
\end{tabular}

Tabla 5. Resultados de grasa total por método combinado.

De acuerdo a estos resultados, se observó que no existe una clara diferencia entre los valores del contenido de grasa para el tipo de producto en cuestión. Lo anterior es debido posiblemente a que las proteínas presentes en la muestra fueron desnaturalizadas en el proceso de cocción al que fue sometida durante la esterilización para su enlatado.

Respecto al nombre del mensurando, basados en que las definiciones de "grasa" van desde: "mezcla de ésteres resultantes de la combinación de glicerina con los ácidos grasos", hasta "extracto etéreo que se obtiene cuando la muestra es sometida a extracción, refiriéndose como 'extracto etéreo' al conjunto de las sustancias extraídas que incluyen, además de los ésteres de los ácidos grasos con el glicerol, a los fosfolípidos, lecitinas, esteroles, ceras, ácidos grasos libres, etc." (Egan et al., 1987), y que "grasa" es también el término que se emplea para aquellas "mezclas de lipidos que son sólidas o semisólidas a temperatura ambiente” (Badui, 1981), se acordó reportar en el certificado el contenido de "extracto etéreo" o "grasa", sin considerar si es el libre o total, o el método de extracción usado.

Con el fin de asignar el valor certificado, se compararon los valores estadísticos de promedio ponderado (Duewer, [s.d.]), el promedio aritmético (EURACHEM, CITAC, 2000; BIPM, 1995) y mediana
(Müller, 2000), con su respectiva incertidumbre asociada.

De acuerdo a los datos de la Tabla 6, tanto los valores como las incertidumbres son comparables, sin embargo se propone emplear como incertidumbre de este ejercicio dos veces la desviación estándar de la media de los participantes. Las incertidumbres evaluadas para estos casos fueron comprobadas mediante el uso de programas estadísticos para cálculo de valores ponderados (Duewer, [s.d.]).

En cuanto a los minerales, las nuevas mediciones reportadas para los analitos de sodio y potasio resultaron más apropiadas para la asignación como valores de referencia, ya que las desviaciones estándar relativas cumplían con los valores meta del proyecto.

No obstante, considerando que la emisión de un valor certificado obtenido mediante el uso de dos métodos de principios diferentes es una práctica aceptada por la Guía ISO 34 (EURACHEM; CITAC, 2000), el laboratorio coordinador propuso que se combinaran los resultados por HPLC e ICP-AES (ambos medidos por el mismo instituto y que corresponden a los resultados de los laboratorios 8 y 9 de las Figuras 4 y 6), y el valor resultante se tomó como valor final. En las Tablas 7 y 8 se detalla cada uno de los valores considerados para generar el valor combinado de sodio y potasio, respectivamente.

\begin{tabular}{|c|c|c|c|c|}
\hline \multirow{2}{*}{ Analito } & & Valor Certificado & $\begin{array}{c}\text { (Incertidumbre expandida } \\
\mathbf{k}=\mathbf{2})\end{array}$ & Unidad \\
\hline \multirow{3}{*}{ Nitrógeno } & Media ponderada & 1,994 & 0,052 & $\mathrm{~g} / 100 \mathrm{~g}$ \\
\cline { 2 - 6 } & Media aritmética & 1,991 & 0,07 & 2,61 \\
\cline { 2 - 6 } & Mediana & 1,988 & 0,061 & 3,51 \\
\hline \multirow{3}{*}{ Grasa } & Media ponderada & 20,19 & 0,43 & $\mathrm{~g} / 100 \mathrm{~g}$ \\
\cline { 2 - 6 } & Media aritmética & 20,16 & 0,53 & 2,13 \\
\cline { 2 - 5 } & Mediana & 20,23 & 0,43 & 2,63 \\
\hline
\end{tabular}

Tabla 6. Valores estadísticos y sus respectivas incertidumbres.

\begin{tabular}{|l|c|c|c|c|}
\hline Técnica & Fracción de masa & U (k=2) & Unidades \\
\hline HPLC & 7848 & 223 & $\mathrm{mg} / \mathrm{kg}$ & 2,85 \\
\hline ICP-AES & 7868 & 146 & $\mathrm{mg} / \mathrm{kg}$ & 1,86 \\
\hline Combinación & 7858 & 267 & $\mathrm{mg} / \mathrm{kg}$ & 3,40 \\
\hline
\end{tabular}

Tabla 7. Valores obtenidos para el contenido de sodio en el candidato a material de referencia de carne.

\begin{tabular}{|l|c|c|c|c|}
\hline Técnica & Fracción de masa & U (k=2) & Unidades \\
\hline HPLC & 1924 & 121 & $\mathrm{mg} / \mathrm{kg}$ & 6,31 \\
\hline ICP-AES & 1943 & 33 & $\mathrm{mg} / \mathrm{kg}$ & 1,68 \\
\hline Combinación & 1934 & 63 & $\mathrm{mg} / \mathrm{kg}$ & 3,26 \\
\hline
\end{tabular}

Tabla 8. Valores obtenidos para el contenido de potasio en el candidato a material de referencia de carne. 


\section{Acciones de mejora}

Dentro de las acciones de mejora que se realizaron durante el proceso para poder concluir con éxito la etapa de certificación del material de carne, se encuentran:

- Armonización de métodos. La estandarización de condiciones de medición permitió a los laboratorios implementar y/o eliminar etapas en sus procedimientos, y así se lograron resultados consistentes con los del resto del grupo.

- Para la determinación de nitrógeno los resultados mejoraron considerablemente al fijar y/o establecer la normalidad del ácido clorhídrico que se utilizó durante el procedimiento de titulación. Adicionalmente, se puso énfasis especial en que los participantes tomaran conciencia de que dicho proceso mejora al usar potenciómetros o voltímetros.

- Para la determinación de grasa el análisis de los resultados según la técnica usada permitió enfatizar acciones importantes, como el secado previo de la muestra durante $5-6 \mathrm{~h}$ a $95-100{ }^{\circ} \mathrm{C}$, debido a su alto contenido de agua (64\%), lo que evitó que los lípidos se liguen de esta forma a proteínas y carbohidratos; la utilización de mezcla de éteres en lugar de uno en particular; el incremento de tiempo de extracción de 4 a 8 horas, etcétera.

- Para minerales la medición de potasio se favoreció al establecer el modo emisión, así como el uso de supresores, lo cual permitió obtener valores menos dispersos. En cuanto a la digestión, fue posible establecer la temperatura de calcinación apropiada para evitar cualquier posible pérdida de los analitos.

- Revisión de resultados. La revisión de resultados, en la que se consideró tanto la dispersión entre muestras como el sesgo de la medición, dio a los laboratorios la pauta para promover programas y cursos de capacitación constantes entre su personal, y al repetir las mediciones los valores obtenidos se observaron claramente más consistentes.

- Concientización del uso de materiales de referencia usados como controles de medición.

- Programas de capacitación técnica. Incluyen intercambios entre los institutos participantes para aprender y/o complementar los conocimientos en la aplicación de las metodologías utilizadas.

\section{Conclusiones}

Se ha mostrado que la calidad de medición bajo el esquema de colaboración es decisiva para el proceso de certificación de materiales de referencia, ya que la contribución de cada participante es crítica para que se puedan lograr los valores certificados con incertidumbres asociadas razonables para la aplicación. Sin embargo, para poder logarlo, es fundamental demostrar competencia de medición.

Mediante este estudio se comprobó que la utilización de métodos armonizados puede sustentar la emisión de valores congruentes aun entre diferentes regiones y legislaciones. Esto permite lograr la generación de materiales de referencia útiles y al alcance de un mayor sector de la industria alimenticia.

En este caso, para los parámetros dependientes de procedimientos operacionales como nitrógeno y grasa se pueden adoptar los valores de consenso, una vez evaluada la consistencia de los resultados; no obstante, para los parámetros independientes de método es necesario emplear métodos de alta jerarquía metrológica, o varios métodos de principios de medición independientes con la competencia apropiada para asignar valores de referencia confiables.

En las Tablas 9 y 10 se presentan los valores certificados y las incertidumbres que fueron adoptadas por los laboratorios para el lote de carne de res tipo paté.

\begin{tabular}{|l|c|c|c|c|}
\hline Analito & Valor de referencia (mediana) & $\begin{array}{c}\text { Incertidumbre (2 veces la } \\
\text { Desviación estándar de la media) }\end{array}$ & Unidades & Urel (\%) \\
\hline Nitrógeno & 1,988 & 0,078 & $\mathrm{~g} / 100 \mathrm{~g}$ & 3,65 \\
\hline Grasa & 20,23 & 0,58 & $\mathrm{~g} / 100 \mathrm{~g}$ & 2,30 \\
\hline
\end{tabular}

Tabla 9. Valores certificados obtenidos para los analitos de nitrógeno y grasa.

\begin{tabular}{|l|c|c|c|c|}
\hline Analito & Valor de referencia combinado & Incertidumbre $(\boldsymbol{k}=\mathbf{2})$ & Unidades & Urel (\%) \\
\hline $\mathbf{N a}$ & 7858 & 267 & $\mathrm{mg} / \mathrm{kg}$ & 3,40 \\
\hline $\mathbf{K}$ & 1934 & 63 & $\mathrm{mg} / \mathrm{kg}$ & 3,26 \\
\hline
\end{tabular}

Tabla 10. Valores certificados obtenidos para los analitos sodio y potasio. 


\begin{tabular}{|c|c|c|c|c|c|c|c|c|}
\hline \multirow[b]{2}{*}{ País } & \multirow[b]{2}{*}{ Método } & \multirow{2}{*}{$\begin{array}{c}\text { Peso } \\
\text { muestra }\end{array}$} & \multicolumn{3}{|c|}{ Digestión } & \multirow{2}{*}{$\frac{\text { Destilación }}{\text { Equipo }}$} & \multicolumn{2}{|c|}{ Titulación } \\
\hline & & & Equipo & Tiempo & Temperatura & & Instrumento & $\begin{array}{l}\text { Determinación del } \\
\text { punto de inflexión }\end{array}$ \\
\hline Argentina & AOAC $981.10(2005)$ & $2,0 \mathrm{~g}$ & Bucchi & $100 \mathrm{~min}$ & $450^{\circ} \mathrm{C}$ & $\begin{array}{c}\text { Bucchi } \\
\text { (automático) }\end{array}$ & Bureta & cambio de color \\
\hline Bolivia & Macro kejldahl & $2,0 \mathrm{~g}$ & $\begin{array}{c}\text { Digestor } \\
\text { macrokejdahl }\end{array}$ & De 1,5 a $2 \mathrm{~h}$ & Nivel 6 & $\begin{array}{c}\text { Destilador } \\
\text { macrokejdahl } \\
\text { (manual) }\end{array}$ & Bureta clase A & cambio de color \\
\hline Brasil & $\begin{array}{l}\text { Horwitz, W } \\
\text { AOAC, } 18 \text { ed }\end{array}$ & $1,5 \mathrm{~g}$ & $\begin{array}{c}\text { TE } 008 / 50-04 \\
\text { TECNAL }\end{array}$ & $150 \mathrm{~min}$ & ------ & $\begin{array}{c}\text { Kjeltec Sistem } \\
1026 \text { Distiling }\end{array}$ & Bureta BUT 0384 & cambio de color \\
\hline Colombia & $\begin{array}{l}\text { AOAC } 981.10 \\
\quad(2005)\end{array}$ & $2 \mathrm{~g}$ & $\begin{array}{c}\text { Digestor } \\
\text { Bucchi } 435\end{array}$ & $3 \mathrm{~h}$ & $\begin{array}{c}\text { Nivel máximo } \\
(10)\end{array}$ & $\begin{array}{c}\text { Destilador } \\
\text { Bucchi } \\
323 \text { (automático) }\end{array}$ & $\begin{array}{l}\text { Titulador Metrohm } \\
702 \text { SM Titrino }\end{array}$ & automático \\
\hline Ecuador & AOAC 960.52 & 0,1 a $0,3 \mathrm{~g}$ & Labconco & $1,5 \mathrm{~h}$ & Nivel máximo & $\begin{array}{l}\text { Labconco } \\
\text { manual }\end{array}$ & Bureta $10 \mathrm{ml} ; 0,04 \mathrm{ml}$ & ph 4,6 \\
\hline Jamaica & Kjeldahl & $1 \mathrm{~g}$ & $\begin{array}{c}\text { Foss } 2020 \\
\text { digester }\end{array}$ & $2 \mathrm{~h}$ & $440^{\circ} \mathrm{C}$ & $\begin{array}{c}\text { Foss } 2020 \\
\text { digester } \\
\text { (automático) }\end{array}$ & Kjeltec 2300 & cambio de color \\
\hline México & Kjeldahl & $1 \mathrm{~g}$ & Bucchi & $2 \mathrm{~h}$ & $380-450^{\circ} \mathrm{C}$ & $\begin{array}{c}\text { Bucchi } \\
\text { (semiautomático) }\end{array}$ & Bureta clase $\mathrm{A}$ & cambio de voltaje \\
\hline Perú - SENASA & Kjeldahl & $0,5 \mathrm{~g}$ & $\begin{array}{c}\text { Digestor } \\
\text { DIGIPREP HT250 }\end{array}$ & $2 \mathrm{~h}$ & $400{ }^{\circ} \mathrm{C}$ & $\begin{array}{c}\text { Digestor } \\
\text { DIGIPREP 200 } \\
\text { (automático) }\end{array}$ & Bureta de $25 \mathrm{~mL}$ & cambio de color \\
\hline Perú - INDECOPI & Kjeldahl & $0,5 \mathrm{~g}$ & Bucchi & $2 \mathrm{~h}$ & $400{ }^{\circ} \mathrm{C}$ & $\begin{array}{c}\text { Bucchi } \\
\text { (automático) }\end{array}$ & Bureta & cambio de color \\
\hline Uruguay & ISO 937:1978 & $1 \mathrm{~g}$ & Gerhardt & $\begin{array}{c}95 \text { min, } \\
\text { previa } \\
\text { clarificación }\end{array}$ & $400^{\circ} \mathrm{C}$ & $\begin{array}{l}\text { Gerhardt } \\
\text { (manual) }\end{array}$ & Bureta & ----- \\
\hline
\end{tabular}

Tabla 11. Diferencias en las metodologías para el análisis de nitrógeno.

\begin{tabular}{|c|c|c|c|c|c|c|c|}
\hline \multirow{2}{*}{ País } & \multirow{2}{*}{ Método } & \multirow{2}{*}{$\begin{array}{c}\text { Peso } \\
\text { muestra }\end{array}$} & \multicolumn{3}{|c|}{ Digestión } & \multicolumn{2}{|c|}{ Evaporación } \\
\hline & & & Agitación & Temperatura & Tiempo & Instrumento & Temperatura \\
\hline Argentina & 960.39 & $5-10 \mathrm{~g}$ & ------ & $65^{\circ} \mathrm{C}$ & $6 \mathrm{~h}$ & Twissel estufa & $102^{\circ} \mathrm{C}$ \\
\hline Bolivia & Soxhlet con hidrólisis previa & $3 \mathrm{~g}$ & ------ & $\begin{array}{c}15 \text { sifoneadas } \\
\text { por hora }\end{array}$ & $6 \mathrm{~h}$ & Baño María & $60 \pm 5^{\circ} \mathrm{C}$ \\
\hline Brasil & $\begin{array}{c}\text { Horwitz, W } \\
\text { AOAC, } 18 \text { ed, } 2005 . \\
\text { (Soxhlet) }\end{array}$ & $10 \mathrm{~g}$ & ------ & $130^{\circ} \mathrm{C}$ & $8 \mathrm{~h}$ & $\begin{array}{l}\text { Baño de } \\
\text { evaporación }\end{array}$ & $60^{\circ} \mathrm{C}$ \\
\hline Ecuador & *CENAM (hidrólisis ácida) & $1,5 \mathrm{~g}$ & Vortex, $60 \mathrm{seg}$ & ----- & $\begin{array}{c}5 \\
\text { extracciones }\end{array}$ & Estufa Heraus & $80^{\circ} \mathrm{C}$ \\
\hline Jamaica & Soxhlet & $1 \mathrm{~g}$ & ----- & $155^{\circ} \mathrm{C}$ & ------ & ------ & ----- \\
\hline México & Hidrólisis ácida & $1-2 g$ & Manual & $70-80^{\circ} \mathrm{C}$ & $40 \mathrm{~min}$ & Estufa & $80^{\circ} \mathrm{C}$ por $8 \mathrm{~h}$ \\
\hline Perú - SENASA & $\begin{array}{c}\text { AOAC } 2003.06 \text { (Soxhlet con } \\
\text { hidrólisis previa) }\end{array}$ & $1 \mathrm{~g}$ & ------ & $180^{\circ} \mathrm{C}$ & $2 \mathrm{~h}$ & Aparato de extracción & $180^{\circ} \mathrm{C}$ por $1 \mathrm{~h}$ \\
\hline Perú - INDECOPI & AOAC 2003.06 & $1-2 \mathrm{~g}$ & Manual, $60 \mathrm{seg}$ & ------ & $\begin{array}{c}3 \\
\text { extracciones }\end{array}$ & ----- & $40^{\circ} \mathrm{C}$ por $1 \mathrm{~h}$ \\
\hline Uruguay & ISO 1443:1973 (soxhlet) & $4 \mathrm{~g}$ & ------ por $1 \mathrm{~h}$ & $\begin{array}{c}\text { Adecuada para } \\
\text { obtener } 20 \\
\text { extracciones por } \\
\text { hora }\end{array}$ & $4 \mathrm{~h}$ & Buchi & $75^{\circ} \mathrm{C}$ por $10 \mathrm{~min}$ \\
\hline
\end{tabular}




\begin{tabular}{|c|c|c|c|c|c|c|c|}
\hline \multirow{2}{*}{ País } & \multirow{2}{*}{ Método } & \multirow{2}{*}{$\begin{array}{l}\text { Peso } \\
\text { muestra }\end{array}$} & \multicolumn{3}{|c|}{ Digestión } & \multicolumn{2}{|c|}{ Medición } \\
\hline & & & Equipo & Tiempo & $\begin{array}{c}\text { Temperatura/ } \\
\text { pontencia }\end{array}$ & Instrumento & Calibrante \\
\hline Argentina & Emisión & $0,5 \mathrm{~g}$ & Horno mufla & $16 \mathrm{~h}$ & $550^{\circ} \mathrm{C}$ & Espectro AA & \begin{tabular}{|l|} 
Na trazable NIST \\
$1 \mathrm{~g} / \mathrm{L}$ lote: 735845
\end{tabular} \\
\hline Bolivia & AA (Flama) & $0,2-0,3 \mathrm{~g}$ & Anton Paar Microwave & $40 \mathrm{~min}$ & $180^{\circ} \mathrm{C}$ & $\begin{array}{c}\text { Analyst } 700 \text { de Perkin } \\
\text { Elmer }\end{array}$ & $\begin{array}{l}\text { Patrones certificados } \\
\text { de Na Perkin Elmer }\end{array}$ \\
\hline Brasil & AA (Horno de grafito) & $0,5 \mathrm{~g}$ & Anton Paar Microwave & $50 \mathrm{~min}$ & $1400 \mathrm{~W}$ & $\begin{array}{c}\text { Perkin Elmer model } \\
800 \text { atomic absorption } \\
\text { spectrometry with } \\
\text { Zeeman-efect } \\
\text { background correction, } \\
\text { THGA } 800 \text { graphite } \\
\text { furnace and an As- } 800 \\
\text { autosampler }\end{array}$ & SRM-3152a \\
\hline Colombia & $\begin{array}{c}\text { Journal of Food Science, } \\
\text { Vol } 56, \text { no. } 5,1991 . \text { (EAAF) }\end{array}$ & $0,5 \mathrm{~g}$ & Horno de microondas CEM & $14 \mathrm{~min}$ & $1400 \mathrm{~W}$ & $\begin{array}{l}\text { AA Thermo } \\
\text { Elemental }\end{array}$ & $\begin{array}{l}\text { Estándar de sodio } \\
\text { de } 0,5 \mathrm{mg} / \mathrm{L}\end{array}$ \\
\hline Ecuador & $\begin{array}{l}\text { AOAC } 985.35 \\
\text { (EAAF) }\end{array}$ & $1 \mathrm{~g}$ & Mufla & $\begin{array}{l}\text { Hasta } \\
\text { cenizas }\end{array}$ & $500^{\circ} \mathrm{C}$ & $\begin{array}{l}\text { Perkin Elmer. Atomic } \\
\text { absorption spectromery }\end{array}$ & Disolución de $\mathrm{NaCl}$ \\
\hline Jamaica & EA(Calibración externa) & $0,5 \mathrm{~g}$ & Estufa carbolite & $4 \mathrm{~h}$ & $600^{\circ} \mathrm{C}$ & $\begin{array}{c}\text { Flame atomic } \\
\text { emmission spectrometry }\end{array}$ & Estándar de $\mathrm{Na}$ \\
\hline México - CLAR & CLAR (estándar interno) & $3 \mathrm{~g}$ & Digestor Buchi & $2 \mathrm{~h}$ & $380-450{ }^{\circ} \mathrm{C}$ & $\begin{array}{l}\text { Cromatógrafo de } \\
\text { líquidos WATERS }\end{array}$ & $\begin{array}{l}\mathrm{NaCl} \text {, disoluciones } \\
\mathrm{de} 2 \text { a } 1000 \mathrm{mg} / \mathrm{kg}\end{array}$ \\
\hline México - ICP-AES & CLAR (calibración externa) & $0,5 \mathrm{~g}$ & Horno de microondas MARSx & $100 \min$ & $1400 \mathrm{~W}$ & $\begin{array}{l}\text { ICP-AES } \\
\text { Iris Intrepid }\end{array}$ & $\begin{array}{l}\mathrm{NaCI} \text {, disoluciones } \\
\text { de } 2 \text { a } 1000 \mathrm{mg} / \mathrm{kg}\end{array}$ \\
\hline Perú - SENASA & USDA CLG-TM 3.00 & $0,3-0,6 \mathrm{~g}$ & Microondas & $10 \mathrm{~min}$ & $120^{\circ} \mathrm{C}$ & ICP-MS & $\begin{array}{c}\text { Estándar } \\
\text { multielemental } \\
\text { de } 1000 \mathrm{ppm}\end{array}$ \\
\hline Uruguay & $\begin{array}{l}\text { Emisión atómica } \\
\text { (estándar interno) }\end{array}$ & $0,5 \mathrm{~g}$ & $\begin{array}{l}\text { Microondas Anton Paar } \\
\text { Multiwave } 3000\end{array}$ & -- & --- & ICP-OES/optima 3300 & SRM-3141a \\
\hline
\end{tabular}

Tabla 13. Diferencias en las metodologías para el análisis de sodio.

\begin{tabular}{|c|c|c|c|c|c|c|c|}
\hline \multirow[b]{2}{*}{ País } & \multirow[b]{2}{*}{ Método } & \multirow{2}{*}{$\begin{array}{l}\text { Peso } \\
\text { muestra }\end{array}$} & \multicolumn{3}{|c|}{ Digestión } & \multicolumn{2}{|c|}{ Medición } \\
\hline & & & Equipo & Tiempo & $\begin{array}{c}\text { Temperatura/ } \\
\text { pontencia }\end{array}$ & Instrumento & Calibrante K \\
\hline Argentina & Emisión & $0,5 \mathrm{~g}$ & Horno mufla & $16 \mathrm{~h}$ & $550^{\circ} \mathrm{C}$ & Espectro AA & $\begin{array}{l}\mathrm{K} \text { trazable NIST } \\
1 \mathrm{~g} / \mathrm{L}\end{array}$ \\
\hline Bolivia & AA (Flama) & $0,2-0,3 \mathrm{~g}$ & Anton Paar Microwave & $40 \mathrm{~min}$ & $180^{\circ} \mathrm{C}$ & $\begin{array}{c}\text { Analyst } 700 \text { de Perkin } \\
\text { Elmer }\end{array}$ & $\begin{array}{l}\text { Patrones certificados } \\
\text { de K de Perkin Elmer }\end{array}$ \\
\hline Brasil & Emisión atómica & $0,5 \mathrm{~g}$ & Anton Paar Microwave & $50 \mathrm{~min}$ & $1400 \mathrm{~W}$ & $\begin{array}{c}\text { Perkin Elmer model } \\
800 \text { atomic absorption } \\
\text { spectrometry }\end{array}$ & SRM-3141a \\
\hline Colombia & $\begin{array}{l}\text { Journal of Food Science, } \\
\text { Vol } 56, \text { no. } 5,1991 \text {. (EAAF) }\end{array}$ & $0,5 \mathrm{~g}$ & Horno de microondas CEM & $14 \mathrm{~min}$ & $1400 \mathrm{~W}$ & $\begin{array}{l}\text { AA Thermo } \\
\text { Elemental }\end{array}$ & \begin{tabular}{|c|} 
Estándar de potasio \\
de $0,8 \mathrm{mg} / \mathrm{L}$
\end{tabular} \\
\hline Jamaica & EEAF (calibración externa) & $0,5 \mathrm{~g}$ & Estufa carbolite & $4 \mathrm{~h}$ & $600^{\circ} \mathrm{C}$ & $\begin{array}{l}\text { Flame atomic } \\
\text { emmission } \\
\text { spectrometry }\end{array}$ & Estándar de $\mathrm{K}$ \\
\hline México - CLAR & CLAR (estándar interno) & $3 \mathrm{~g}$ & Digestor Buchi & $2 \mathrm{~h}$ & $380-450^{\circ} \mathrm{C}$ & $\begin{array}{l}\text { Cromatógrafo de } \\
\text { líquidos WATERS }\end{array}$ & $\begin{array}{l}\text { KCI, disoluciones } \\
\text { de } 2 \text { a } 1000 \mathrm{mg} / \mathrm{kg}\end{array}$ \\
\hline México - ICP-AES & $\begin{array}{c}\text { ICP-AES } \\
\text { (calibración externa) }\end{array}$ & $0,5 \mathrm{~g}$ & Horno de microondas MARSx & $100 \mathrm{~min}$ & $1400 \mathrm{~W}$ & $\begin{array}{l}\text { ICP-AES } \\
\text { Iris Intrepid }\end{array}$ & $\begin{array}{l}\text { KCI, disoluciones } \\
\text { de } 2 \text { a } 1000 \mathrm{mg} / \mathrm{kg}\end{array}$ \\
\hline Perú - SENASA & USDA CLG-TM 3.00 & $0,3-0,6 \mathrm{~g}$ & Microondas & $10 \mathrm{~min}$ & $120^{\circ} \mathrm{C}$ & ICP-MS & $\begin{array}{c}\text { Estándar } \\
\text { multielemental } \\
\text { de } 1000 \mathrm{ppm}\end{array}$ \\
\hline Uruguay & ICP-AES (estándar interno) & $0,5 \mathrm{~g}$ & $\begin{array}{l}\text { Microondas Anton Paar } \\
\text { Multiwave } 3000\end{array}$ & --- & --- & ICP-OES/optima 3300 & SRM-3141a \\
\hline
\end{tabular}

Tabla 14. Diferencias en las metodologías para el análisis de potasio. 


\section{Reconocimientos}

A la Organización de los Estados Americanos por el apoyo del proyecto SEDI/AICD/AE/033/08 "Colaboración para Soportar con Metrología Química la Evaluación de la Calidad e Inocuidad de los Productos del Sector Agroalimentario", y a la Dra. Mariana Arce Osuna por su implantación. A la I.Q. Judith García Escalante por su apoyo logístico de coordinación. A los participantes en las mediciones en esta etapa. A los coordinadores de los institutos participantes: Celia Puglisi, Juan Carlos Castillo, Mabel Delgado, Janaina Marques, Carlos Eduardo Porras, Mónica Gualotuña, James Kerr, Dwight Random, Yoshito Mitani, José Dajes, Elizabeth Ferreira, Claudia Santo.

\section{Referencias}

- BADUI, Dergal S. Química de los alimentos. México: Alhambra Mexicana, 1981.

- BIPM; IEC; IFCC; ISO; IUPAC; IUPAP; OIML. Guide to the expression of uncertainty in measurement. Geneva: International Organization for Standardization, 1995.

- DUEWER, D. L. A robust approach for the determination of CCQM key comparison reference values and uncertainties [En línea]. Gaithersburg: NIST, [s.d.]. [Consulta: 25 de octubre de 2010]. Disponible en: http://www.bipm.net/cc/CCQM/Allowed/10/ CCQM04-15.pdf

- EGAN, H.; KIRK, R.S.; SAWYER, R. Análisis químico de alimentos de Pearson. México: Compañía Editorial Continental, 1987.

- EURACHEM; CITAC. Quantifying uncertainty in analytical measurement. 2da. ed. [s.1.]: EURACHEM, 2000. ISBN 0948926 155.

- MÜLLER, J. W. Possible advantages of a robust evaluation of comparisons. En: Journal of Research at the National Institute of Standards and Technology. 2000, 105:551.

- SOCIEDAD MEXICANA DE NORMALIZACIÓN Y CERTIFICACIÓN S.C. (NORMEX). (México). NMX-F-615NORMEX-2004: Alimentos - Determinación de extracto etéreo. México: NORMEX, 2004. 